\title{
Is nevtral NEUTRAL? Visual similarity effects in the early phases of written-word recognition
}

\author{
${\text { Ana } \text { Marcet }^{1} \cdot \text { Manuel Perea }}^{1}$ \\ Published online: 21 November 2016 \\ (C) Psychonomic Society, Inc. 2016
}

\begin{abstract}
For simplicity, contemporary models of written-word recognition and reading have unspecified feature/letter levels - they predict that the visually similar substituted-letter nonword PEQPLE is as effective at activating the word PEOPLE as the visually dissimilar substituted-letter nonword PEYPLE. Previous empirical evidence on the effects of visual similarly across letters during written-word recognition is scarce and nonconclusive. To examine whether visual similarity across letters plays a role early in word processing, we conducted two masked priming lexical decision experiments (stimulus-onset asynchrony $=50 \mathrm{~ms}$ ). The substituted-letter primes were visually very similar to the target letters $(u / v$ in Experiment 1 and $i / j$ in Experiment 2; e.g., nevtral-NEUTRAL). For comparison purposes, we included an identity prime condition (neutral-NEUTRAL) and a dissimilar-letter prime condition (neztral-NEUTRAL). Results showed that the similarletter prime condition produced faster word identification times than the dissimilar-letter prime condition. We discuss how models of written-word recognition should be amended to capture visual similarity effects across letters.
\end{abstract}

Keywords Visual similarity · Masked priming - Lexical access

Ana Marcet

Ana.Marcet@uv.es

1 Departamento de Metodología and ERI-Lectura, Universitat de València, Av. Blasco Ibáñez, 21, 46010 Valencia, Spain
Contemporary models of written-word recognition and reading in the Roman alphabet share the assumption that lexical access takes place on the basis of case-invariant abstract letter representations that are attained early in processing (Grainger, Dufau, \& Ziegler, 2016). For simplicity's sake, these models assume a minimal/null role of visual similarity across letters in lexical access. Using the default parameters in the interactive activation model (Rumelhart \& McClelland, 1982) and its successors (e.g., spatial coding model; Davis, 2010), the visually similar substituted-letter prime PEQPLE is as effective at activating the word PEOPLE as the visually dissimilar substituted-letter prime PEYPLE (i.e., each condition yielded 60 processing cycles in masked priming lexical decision using Davis's, 2010, simulator) - note that $\mathrm{O}$ and $\mathrm{Q}$ share all features but one at the feature-letter level ( and $Y$ do not share any features $(Y, Y)$. Likewise, other leading models posit that all letters are equally confusable (Bayesian reader model: Norris, 2006; Rationale model of eye movements in reading: Bicknell \& Levy, 2010) so they would also predict similar word identification times for PEQPLE-PEOPLE and PEYPLE-PEOPLE.

Nonetheless, if we assume that it takes time for the cognitive system to encode letter identity (or letter position), visual similarity across letters should have an impact in the early phases of word processing. Clearly, if PEQPLE-PEOPLE produces faster word recognition times than PEYPLE-PEOPLE, modelers should make an effort to develop in greater depth the underpinnings of the links between the feature and letter levels (i.e., this finding could be used as a benchmark for what is there to simulate). An analogy with letter position coding is relevant here: The slot-coding schemes in the interactive activation model and the Bayesian reader were admittedly oversimplifications (see Norris, 2006, p. 346; Rumelhart \& McClelland, 1982, p. 89). The 
literature on letter transposition effects in past decades ruled out these schemes and led to more sophisticated accounts of letter position coding (e.g., spatial coding model: Davis, 2010; noisy Bayesian reader model: Norris, Kinoshita, \& van Casteren, 2010).

Visual similarity effects have been reported with letter-like digits and letter-like symbols with the masked priming technique. For example, Perea, Duñabeitia, and Carreiras (2008) found that lexical decision times for a target word like MATERIAL were faster when preceded by a visually similar digit prime (M473RI4L; i.e., a prime that included letter-like digits such as $4=\mathrm{A}, 3$ $=\mathrm{E}$ or $7=\mathrm{T}$ ) or a visually similar symbol prime (e.g., $\mathrm{M} \triangle \mathrm{T} € \mathrm{R} ! \Delta \mathrm{L})$ than when preceded by a control prime (M568RI2L or MøT\%R?口L; see Kinoshita \& Lagoutaris, 2010; Lien, Allen, \& Martin, 2014, for converging evidence). Furthermore, visually similar digit/ symbol primes were nearly as facilitative as identity primes (Perea et al., 2008).

As visual similarity with letter-like digits/symbols plays a role early during written-word identification, one would expect a parallel effect with visually similar substituted-letter primes. Indeed, a number of experiments on letter identification have obtained effects of visual similarity (e.g., the letters $B$ and $R$ are more confusable than $B$ and $G$; for review, see Mueller \& Weidemann, 2012). However, the empirical evidence in the word recognition literature is scarce and nonconclusive. In a single-presentation lexical decision task, Perea and Panadero (2014) found more "word" responses to viotin-type nonwords (i.e., one-letter different nonwords that looked visually similar to their base word [violin]) than to viocin-type nonwords in individuals with dyslexia, but the effect did not occur in normally reading individuals. To study in detail the effects of visual similarity early in processing, masked priming is a better option than a single-presentation paradigm. Kinoshita, Robidoux, Mills, and Norris (2013) conducted a masked priming lexical decision experiment in which a target word (e.g., abandon) could be preceded by (a) a visually similar digit prime $(484 \mathrm{NDON} ; 4$ is visually similar to $A$ and 8 is visually similar to $B$ ); (b) a visually dissimilar digit prime $(676 \mathrm{NDON})$; (c) a visually similar letter prime (HRHNDON; $H$ is visually similar to $A$ and $R$ is visually similar to $B$ ); or (d) a visually dissimilar letter prime (DWDNDON) - they also included an identity priming condition (ABANDON) and an unrelated condition (PRODUCT). As in prior research, they found faster word identification times for $484 \mathrm{NDON}$-abandon than for $676 \mathrm{NDON}$-abandonthe word identification times for 484NDON-abandon were nearly the same as those for ABANDON-abandon. But the critical finding was that they failed to find a significant difference between HRHNDON-abandon and DWDNDON-abandon.

To explain the null visual similarity effect for substituted-letter primes, Kinoshita et al. (2013) suggested that "the letter representations $\mathrm{A}$ and $\mathrm{H}$ may be connected by a bidirectional inhibitory link, such that the activation of one drives down the activation of the other" (p. 828) However, a closer look at the priming effects reported by Kinoshita et al. (2013) reveals an 8ms advantage of HRHNDON-abandon over DWDNDON-abandon $(p=.0982)$-there were only 20 items/condition $(N=37 ; 740$ data points in each cell). In a recent simulation study, Stevens and Brysbaert (2016) claimed that "a properly powered experiment requires at least 1,600 word observations per condition for the orthographic priming study" (p. 2). Thus, it may be premature to conclude that visual similarity across letters does not play a role early in word processing.

The goal of the current masked priming lexical decision experiments was to examine whether visual similarity across letters plays a role in the early phases of written-word recognition using a large number of data points per condition $(2,160 ; 80$ items/condition; $N=27$ in each experiment). Each target word was briefly preceded by (a) a lowercase identity prime; (b) a visually similar substituted-letter prime; or (c) a visually dissimilar substituted-letter prime. In Experiment 1, we used two critical letters $(u$ and $v)$ that had a high degree of estimated visual similarity: 4.93 in a 7-point Likert scale (Simpson, Mousikou, Montoya, \& Defior, 2012) (e.g., neutral-NEUTRAL vs. nevtral-NEUTRAL vs. neztralNEUTRAL). Experiment 2 was designed to replicate Experiment 1 with a different set of words; furthermore, the critical letters ( $i$ and $j$ ) had an even greater degree of visual similarity (5.17 out of 7; Simpson et al., 2012).

The predictions are clear. If each letter only activates its own representation early in word processing, possibly via bidirectional inhibitory links across letters (e.g., the letter $j$, but not $i$ or $o$, would activate the abstract representation of $j$ ), one would expect a similar advantage of the identity condition over both the visually similar and visually dissimilar letter conditions. This outcome would not require any major modifications in contemporary models of written-word recognition. Alternatively, if visual letter similarity plays a role in the early phases of written-word recognition (e.g., the letter $j$, and to some degree the visually similar letter $i$, would activate the abstract representation of $j$ ), one would expect an advantage of the visually similar letter condition over the visually dissimilar letter condition. This result would require more elaborated accounts of the feature/letter levels in models of written-word recognition. 


\section{Experiment 1}

\section{Method}

\section{Participants}

The participants were 27 undergraduate students from the Universitat de València. All of them were native speakers of Spanish with normal/corrected-to-normal vision. Written informed consent was obtained from all participants.

\section{Materials}

We selected two hundred and forty Spanish words from the EsPal subtitle database (Duchon, Perea, SebastiánGallés, Martí, \& Carreiras 2013). The average Zipf frequency was 3.67 (range: 1.71-5.91), the average number of letters was 7.5 (range: 5-11), and the average Levenshtein distance (OLD20) was 2.1 (range: 1.24.3). All words had the letters $u$ or $v$ in an internal position (e.g., NEUTRAL; CAVERNA [cavern]). Target words were presented in capital letters and were preceded by (a) an identity prime in lowercase (identity condition; neutral-NEUTRAL; caverna-CAVERNA); (b) a nonword prime in lowercase, in which the letter $u / v$ from the base word was replaced by $v / u$ (visually similar letter prime condition; nevtral-NEUTRAL; cauernaCAVERNA); or (c) a nonword prime in lowercase, in which the letter $u / v$ from the base word was replaced by a visually dissimilar letter-each keeping a neutral form (i.e., letters with no ascenders/descenders) and the same consonant/vowel status as the visually similar letter prime (visually dissimilar letter prime condition; neztral-NEUTRAL; caoerna-CAVERNA). Likewise, we created 240 nonwords, with the same length as words, using Wuggy (Keuleers \& Brysbaert, 2010). All nonwords had the letter $u$ or $v$ in an internal position (e.g., CARCURA; OLCLIVO) and the same primetarget manipulation as that for the words (i.e., an identity condition, a visually similar letter prime condition, and a visually dissimilar letter prime condition). To counterbalance the prime-target pairs across conditions, we created three lists in a Latin square manner. The words/nonwords are available at http://www.uv. es/amarhe5/VisSim.pdf

\section{Procedure}

Participants were tested individually in a silent room. We used DMDX to present the stimuli and register the responses (Forster \& Forster, 2003). Participants were informed that, in each trial, they would be presented with a letter string that could form (or not) a word in
Spanish. Their task was to press, as quickly and as accurately as possible, the key for si [yes] or no. The sequence of each trial was as follows: (1) a pattern mask composed of a series of \#'s was presented in the center of a CRT screen for $500 \mathrm{~ms}$ (the length of the mask was the same as the length of the prime/target); (2) a prime stimulus (in lowercase) replaced the mask in the same spatial location for $50 \mathrm{~ms}$; and (3) a target (in uppercase) replaced the prime in the same spatial location until the participant responded (or $2 \mathrm{~s}$ had elapsed). All stimuli were presented in a fixed-width font (14-pt Consolas). Stimulus presentation was randomized for each participant. Sixteen practice trials preceded the 240 experimental trials. The whole session lasted for approximately 18-20 minutes.

\section{Results and discussion}

Error responses (6.0\% for words; $3.6 \%$ for nonwords) and correct response times (RTs) shorter than $250 \mathrm{~ms}$ $(0.0 \%$ of the data) were omitted from the latency analyses. The mean RTs for correct responses and accuracy are displayed in Table 1. As in the Kinoshita et al. (2013) experiment, we focused on the word targetsnote that masked form priming effects for nonwords tend to be unreliable.

To examine the effect of type of prime (identity [ID], similar letter [SIM], dissimilar letter [DIS]), we conducted linear mixed-effects models using the lme 4 and lmerTest $\mathrm{R}$ packages. Because of the positive skew of the RT data, we employed $-1,000 /$ RT (instead of raw RT) as the dependent variable in the latency analyses. There were 6,247 observations. We coded the levels of type of prime so that the model would test the two comparisons of interest (i.e., SIM vs. DIS and ID vs. SIM). The model included random intercepts for subjects and items as well as the by-subject and by-items random slopes for type of prime (i.e., the maximal random effects structure). The analyses on the accuracy data were modeled using the glmer function in $\mathrm{R}$ (lme4 package), where the accuracy data were coded as binary values $(1=$ correct, $0=$ incorrect $){ }^{1}$

The statistical analyses of the word identification times showed an 11-ms advantage of the SIM priming condition over the DIS priming condition (613 vs. $624 \mathrm{~ms}$, respectively), $t=2.83, p=.005$. In addition, there was a significant $10-\mathrm{ms}$ advantage of the ID priming condition over the SIM priming condition, $t=-2.53, p=.012$.

\footnotetext{
${ }^{1}$ In both experiments, the pattern of significant effects was the same in standard ANOVAs in which the means were aggregated over items/ subjects.
} 
Table 1 Mean lexical decision times (in ms) and accuracy (in parentheses) for words and nonwords in Experiment 1

\begin{tabular}{llll}
\hline & Identity & Similar letter & Dissimilar letter \\
\hline Words & $603(.971)$ & $613(.968)$ & $624(.954)$ \\
Nonwords & $720(.932)$ & $722(.944)$ & $726(.943)$ \\
\hline
\end{tabular}

The statistical analyses of the accuracy showed higher accuracy in the SIM priming condition than in the DIS priming condition (0.968 vs. 0.953 , respectively), $z=-2.45, p=.014$, whereas there were no differences in accuracy between the ID and SIM priming conditions ( 0.971 vs. 0.968 , respectively), $|z|<1$.

The results are straightforward: We found a significant 11-ms advantage of the SIM condition over the DIS condition-this effect was virtually the same for $u \rightarrow v$ and $v u \rightarrow v$ substitutions.

This finding suggests that visual similarity across letters does play a role in the early phases of written-word recognition. To reach firmer conclusions, it is important to replicate the experiment with another set of stimuli and critical letters. To that end, we designed Experiment 2. This experiment was parallel to Experiment 1, except that we employed the letters $i / j$ instead of the letters $u / v$ as the visually similar letters.

\section{Experiment 2}

\section{Method}

\section{Participants}

Twenty-seven students from the same population as in Experiment 1 took part in the experiment. None of them had participated in Experiment 1.

\section{Materials}

We obtained a set of 240 Spanish words from the EsPal subtitle database (Duchon et al., 2013). The average Zipf frequency was 4.08 (range: 3.33-5.50), the average number of letters was 7.6 (range: 5-11), and the average Levenshtein distance was 2.2 (range: 1.3-4.3). All words had the letters $i$ or $j$ in an internal position (e.g., DENTISTA [dentist]; PASAJERO [passenger]). For each target word, we created three primes: (1) an identity prime (dentista-DENTISTA; pasajero-PASAJERO); (2) a visually similar letter prime (dentjsta-DENTISTA; pasaiero-PASAJERO); (3) a visually dissimilar letter prime (dentgsta-DENTISTA; pasauero-PASAJERO). We also created 240 nonwords in the same manner as in Experiment 1. All nonwords had the letter $i$ or $j$ in an internal position (e.g., BESTINDA; MOMAJERA). The manipulation for the nonwords was the same as that for the words. The set of words/nonwords is available at http://www.uv.es/amarhe5/VisSim.pdf

\section{Procedure}

The procedure was the same as in Experiment 1.

\section{Results and discussion}

Incorrect responses $(3.58 \%$ for words and $6.03 \%$ for nonwords) and correct response times (RTs) shorter than $250 \mathrm{~ms}$ (less than $0.02 \%$ ) were excluded from the latency analyses. The mean RTs for correct responses and accuracy are displayed in Table 2.

The statistical analyses were parallel to those in Experiment 1. That is, we examined the effect of type of prime (identity [ID], similar letter [SIM], dissimilar letter [DIS]) using linear mixed-effects models. There were 6,212 observations in the RT data. The model for the RT data included random intercepts for subjects and items as well as the by-subject random slopes for type of prime - the maximal random effects structure model did not converge.

The statistical analyses of the RT data showed a 19ms advantage of the SIM priming condition over the DIS priming condition (606 vs. $625 \mathrm{~ms}$, respectively), $t$ $=5.05, p<.001$, whereas there were no signs of a difference $(<1 \mathrm{~ms})$ between the ID and SIM priming conditions (606 ms in both conditions), $|t|<1$.

The statistical analyses of the accuracy showed parallel results as the latency data: The SIM priming condition was responded to more accurately than the DIS priming condition ( 0.968 vs. 0.954 , respectively), $z=-$ $2.45, p=.015$, whereas there were no signs of a difference between the ID and SIM priming conditions (0.971 vs. 0.968 , respectively), $|z|<1$.

Thus, as in Experiment 1, we found an advantage of the SIM condition over the DIS condition. Furthermore, the SIM condition behaved similarly to

Table 2 Mean lexical decision times (in ms) and accuracy (in parentheses) for words and nonwords in Experiment 2

\begin{tabular}{llll}
\hline & Identity & Similar letter & Dissimilar letter \\
\hline Words & $606(.971)$ & $606(.968)$ & $625(.954)$ \\
Nonwords & $738(.932)$ & $737(.944)$ & $729(.943)$ \\
\hline
\end{tabular}


the ID condition-note that the visual similarity of the critical letter pair was higher than in Experiment 1.

\section{General discussion}

The present masked priming experiments examined, using a large number of data points per condition $(2,160)$, whether visual similarity across letters plays a role during the early phases of written-word recognition. Results showed a sizable advantage of the visually similar letter (SIM) condition over the visually dissimilar letter (DIS) condition: $11 \mathrm{~ms}$ in Experiment 1 (nevtral-NEUTRAL faster than neztral-NEUTRAL) and $19 \mathrm{~ms}$ in Experiment 2 (dentjsta-DENTISTA faster than dentgsta-DENTISTA). This finding is consistent with previous facilitative effects of visual similarity for letter-like digits (e.g., 4 in M4TERI4LMATERIAL) and letter-like symbols (e.g., $\Delta$ in $\mathrm{M} \triangle \mathrm{TERI} \Delta \mathrm{L}-\mathrm{MATERIAL}$ ) in masked priming experiments (Kinoshita \& Lagoutaris, 2010; Lien et al., 2014; Perea et al., 2008). The divergences between the present data and Kinoshita et al.'s (2013) data with substituted-letter primes are more apparent than real. Kinoshita et al. (2013) found an 8-ms advantage of the visually similar letter condition over the visually dissimilar letter condition $(p=.0982)$, with a lower number of data points per condition $(740){ }^{2}$

The current findings have relevant implications for models of written-word recognition and reading. The presence of a masked priming effect of visual similarity with substituted-letter primes implies that there is some degree of ambiguity concerning letter identities in the early phases of word processing (see Norris et al., 2010) and, hence, models of written-word recognition should account for this effect (i.e., it can be used as a benchmark for future simulation studies). Indeed, a number of experiments have shown that word identification times (and eye fixation times) are longer for the word BRUNCH, which has a higher frequency one-letter different neighbor (BRANCH), than for a control word (e.g., BUFFET, which does not have higher frequency neighbors; Grainger, O'Regan, Jacobs, \& Segui, 1989; Slattery 2009; see

\footnotetext{
${ }^{2}$ Some hints in the data suggest that the degree of visual similarity across letters could modulate the effect. When the letter similarity of the critical pair was 4.93 of 7, we found an advantage of the SIM condition over the DIS condition (11 ms) that was accompanied by an advantage of the ID condition over the SIM condition (10 ms). When the visual similarity of the critical pair of letters was higher (5.17), we found a larger advantage of the SIM condition over the DIS condition $(19 \mathrm{~ms})$, whereas there were no signs of a difference between the ID and SIM conditions $(<1 \mathrm{~ms})$. Further research should examine the precise role of visual similarity in this effect.
}

also Segui \& Grainger, 1990, for masked priming evidence). Clearly, if a word's letter identities were perfectly attained in the early phases of word processing, one would not expect neighborhood frequency effects to occur during written-word recognition and reading.

In their implementation of the Bayesian reader model, Norris et al. (2010) acknowledged that the assumption of similar confusability for all letters was "unlikely to be an accurate characterisation of human perception" (p. 347). Similarly, in their model of eye movements in reading, Bicknell and Levy (2010) indicated that this assumption was "ignoring work on letter confusability which could be added to future model revisions" ( $\mathrm{p}$. 1172). The same argument applies to those computational models of written-word recognition that employ the orthographic coding scheme of Rumelhart and McClelland's (1982) interactive-activation model (e.g., spatial coding model; Davis, 2010) - these models assume an unrealistic letter feature level that only incorporates an uppercase font composed of straight lines. As Davis (2010) indicated, future implementations of these models should incorporate a more sophisticated letter coding scheme to encode letter representations from their visual features. Three of the main challenges for modelers are how to specify (1) the most diagnostic visual elements of letters (e.g., lines, curves, intersections, terminations) in the initial phases of word processing (see Blais et al., 2009; Rosa, Perea, \& Enneson, 2016, for discussion); (2) how these visual features are dynamically weighted (see Wiley, Wilson, \& Rapp, 2016) ${ }^{3}$; and (3) how visual information is mapped onto abstract representations (see Grainger et al., 2016). Although a thorough description of these questions would be beyond the scope of this study, it is clear that additional research is needed to help determine the time course of visual similarity effects across letters in during written-word recognition.

In sum, we found an advantage of visually similar substituted-letter primes over visually dissimilar substituted-letter primes in the initial stages of word processing. This finding strongly suggests that future implementations of models of written-word recognition and reading should employ more refined letterfeature and letter levels.

\footnotetext{
${ }^{3}$ In a masked priming lexical decision experiment in Arabic, Perea, Abu Mallouh, Mohammed, Khalifa, and Carreiras (2016) found similar RTs in a substituted-letter priming condition that only differed from the target in the number of diacritical marks (e.g., خفية - حفية) and in a substitutedletter priming condition that differed in letter shape (كفية - حفية). This finding reveals that diacritical marks are encoded very rapidly-note that most letters in Arabic differ only in the number/location of diacritical marks. As Wiley et al. (2016) showed, diacritical marks are the most relevant element to discriminating letters in Arabic.
} 
Authors Notes This research has been partially supported by Grants PSI2014-53444-P and BES-2015-07414 from the Spanish Government. We thank Colin Davis, Sachiko Kinoshita, and an anonymous reviewer for their constructive feedback on a previous version of this article.

\section{References}

Bicknell, K., \& Levy, R. (2010). A rational model of eye movement control in reading. In J. Hajič (Ed.), Proceedings of the 48th Annual Meeting of the Association for Computational Linguistics (pp. 1168-1178). Uppsala, Sweden: Association for Computational Linguistics. doi:10.1037/e520602012-979

Blais, C., Fiset, D., Jolicoeur, P., Arguin, M., Bub, D. N., \& Gosselin, F. (2009). Reading between eye saccades. PLOS ONE, 4(7), e6448. doi:10.1371/journal.pone. 0006448

Davis, C. J. (2010). The spatial coding model of visual word identification. Psychological Review, 117, 713-758. doi:10.1037/a0019738

Duchon, A., Perea, M., Sebastián-Gallés, N., Martí, A., \& Carreiras, M. (2013). EsPal: One-stop shopping for Spanish word properties. Behavior Research Methods, 45, 1246-1258. doi:10.3758/s13428013-0326-1

Forster, K. I., \& Forster, J. C. (2003). DMDX: A Windows display program with millisecond accuracy. Behavior Research Methods, Instruments, \& Computers, 35, 116-124. doi:10.3758/bf03195503

Grainger, J., Dufau, S., \& Ziegler, J. C. (2016). A vision of reading. Trends in Cognitive Sciences, 20, 171-179. doi:10.1016/j. tics.2015.12.008

Grainger, J., O’Regan, J. K., Jacobs, A. M., \& Segui, J. (1989). On the role of competing word units in visual word recognition: The neighborhood frequency effect. Perception \& Psychophysics, 45, 189195. doi:10.3758/bf03210696

Keuleers, E., \& Brysbaert, M. (2010). Wuggy: A multilingual pseudoword generator. Behavior Research Methods, 42, 627-633. doi:10.3758/brm.42.3.627

Kinoshita, S., \& Lagoutaris, S. (2010). Priming by numb3r5 does not involve top-down feedback. Journal of Experimental Psychology: Learning, Memory, and Cognition, 36, 1422-1440. doi:10.1037/a0020609

Kinoshita, S., Robidoux, S., Mills, L., \& Norris, D. (2013). Visual similarity effects on masked priming. Memory \& Cognition, 42, 821833. doi:10.3758/s13421-013-0388-4

Lien, M.-C., Allen, P., \& Martin, N. (2014). Processing visual words with numbers: Electrophysiological evidence for semantic activation. Psychonomic Bulletin and Review, 21, 1056-1066. doi:10.3758/s13423-014-0581-x
Mueller, S. T., \& Weidemann, C. T. (2012). Alphabetic letter identification: Effects of perceivability, similarity, and bias. Acta Psychologica, 139, 19-37. doi:10.1016/j.actpsy.2011.09.014

Norris, D. (2006). The Bayesian reader: Explaining word recognition as an optimal Bayesian decision process. Psychological Review, 113, 327-357. doi:10.1037/0033-295x.113.2.327

Norris, D., Kinoshita, S., \& van Casteren, M. (2010). A stimulus sampling theory of letter identity and order. Journal of Memory and Language, 62, 254-271. doi:10.1016/j.jml.2009.11.002

Perea, M., Abu Mallouh, R., Mohammed, A., Khalifa, B., \& Carreiras, M. (2016). Do diacritical marks play a role at the early stages of word recognition in Arabic? Frontiers in Psychology, 7, 1255. doi:10.3389/fpsyg.2016.01255

Perea, M., Duñabeitia, J. A., \& Carreiras, M. (2008). R34D1NG W0RD5 W1TH NUMB3R5. Journal of Experimental Psychology: Human Perception and Performance, 34, 237-241. doi:10.1037/00961523.34.1.237

Perea, M., \& Panadero, V. (2014). Does viotin activate violin more than viocin? On the use of visual cues during visual-word recognition. Experimental Psychology, 61, 23-29. doi:10.1027/1618-3169/a000223

Rosa, E., Perea, M., \& Enneson, P. (2016). The role of letter features in visual-word recognition: Evidence from a delayed segment technique. Acta Psychologica, 169, 133-142. doi:10.1016/j. actpsy.2016.05.016

Rumelhart, D. E., \& McClelland, J. L. (1982). An interactive activation model of context effects in letter perception: II. The contextual enhancement effect and some tests and extensions of the model. Psychological Review, 89, 60-94. doi:10.1037/0033-295x.89.1.60

Segui, J., \& Grainger, J. (1990). Priming word recognition with orthographic neighbors: Effects of relative prime-target frequency. Journal of Experimental Psychology: Human Perception and Performance, 16, 65-76. doi:10.1037/0096-1523.16.1.65

Simpson, I. C., Mousikou, P., Montoya, J. M., \& Defior, S. (2012). A letter visual-similarity matrix for Latin-based alphabets. Behavior Research Methods, 45, 431-439. doi:10.3758/s13428-012-0271-4

Slattery, T. J. (2009). Word misperception, the neighbor frequency effect, and the role of sentence context: Evidence from eye movements. Journal of Experimental Psychology: Human Perception and Performance, 35, 1969-1975. doi:10.1037/a0016894

Stevens, M. A., \& Brysbaert, M. (2016, January 27). Power in language research. Retrieved from osf.io/d2cye

Wiley, R. W., Wilson, C., \& Rapp, B. (2016). The effects of alphabet and expertise on letter perception. Journal of Experimental Psychology: Human Perception and Performance, 42, 11861203. doi: $10.1037 / \mathrm{xhp} 0000213$ 